\title{
Examining the Benefits of Paternal Involvement and Breaking the Myths Surrounding Fathers
}

Barb Himes, IBCLC

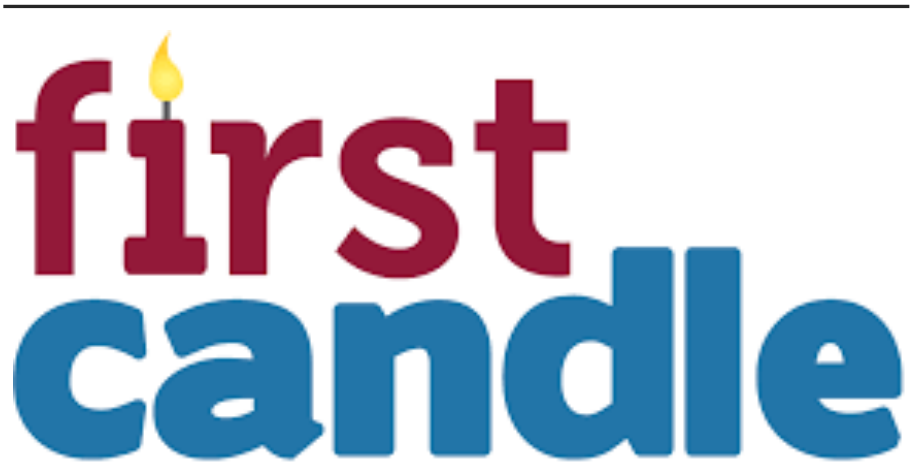

Saving babies. Supporting families.

First Candle's efforts to support families during their most difficult times and provide new answers to help other families avoid the tragedy of the loss of their baby are without parallel.

Sudden Unexplained Infant Death (SUID), which includes Sudden Infant Death Syndrome (SIDS) and accidental suffocation and strangulation in bed (ASSB), remains the leading cause of death for babies one month to one year of age, resulting in 3,600 infant deaths nationwide each year. This is despite the National Institute of Health's Back to Sleep campaign launched in 1994, based on sale sleep guidelines from the American Academy of Pediatrics (AAP), which led to an initial $50 \%$ reduction in the SIDS mortality rate.

That this remains the case is an indication of the complexities involved in maternal and infant health, both behavioral and physical. First Candle was a part of the Back to Sleep campaign collaboration and has made the AAP guidelines -- supine sleep on a firm surface, alone, with no bed-sharing or extraneous bed sharing -- the foundation of its Straight Talk for Infant Safe Sleep training program, working with community agencies and health care providers to educate families about the importance of an infant safe sleep environment.

Not only has the national SIDS death rate not substantially

\section{Introduction}

changed, but SUID rates have also quadrupled since 1984 and are three times greater in Black communities, and research and observation reveal implicit bias in the health care community as a barrier, coupled with cultural and socioeconomic factors that favor bed-sharing and other at-risk sleep environments.

This bias may tend to be maternally focused, but it may also lead practitioners to disregard the potential importance of fathers.

\section{The Value of Paternal Involvement}

Paternal involvement has been shown to have an impact on pregnancy and infant outcomes, (1) including a positive effect on maternal health behaviors and reduced risk of preterm birth, low birth weight, and infant mortality up to one year after birth. But fathers may be overlooked, especially in Black families.

As an illustration, breastfeeding has been associated with a reduction in SIDS deaths (2) and research also indicates that paternal involvement can have a positive effect on breastfeeding, (3) but Black infants are $21 \%$ less likely to have ever been breastfed than any other ethnicity, and only $68 \%$ of Black women try breastfeeding after giving birth, compared to $85.7 \%$ of white women and $84.8 \%$ percent of Hispanic women. In Michigan, for example, $77.3 \%$ of Black women start breastfeeding, and $35.2 \%$ continue to three months.(4)

\section{"Paternal involvement has been shown to} have an impact on pregnancy and infant outcomes, including a positive effect on maternal health behaviors and reduced risk of preterm birth, low birth weight, and infant mortality up to one year after birth. But fathers may be overlooked, especially in Black families."

Peter M. Williams, BPA, CHW, CLC, a Fatherhood Community Health Worker in the Detroit Health Department, is addressing this first-hand, through community outreach regarding the role of fathers and working with fathers themselves.

"There are misconceptions about fathers, especially those in Black

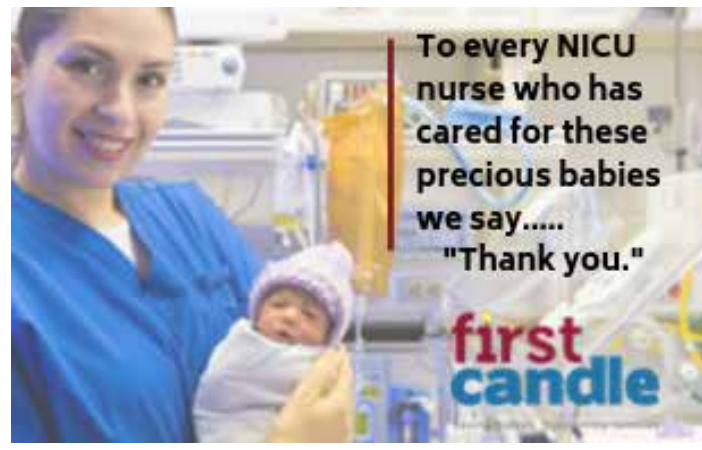

Did you know that premature and low birth weight babies have a $4 \times$ greater risk for SIDS?

At First Candle we're educating parents, grandparents and caregivers about safer sleep to make sure all babies reach their first birthday. Learn more at firstcandle.org 
communities," Williams said. "The birthing process has been traditionally mom-focused, with dads seen as a sort of on the edge of things and maybe not interested in becoming involved."

Williams has conducted focus groups with fathers, where they tell him they want to be involved with the children at the outset, but that they also needed help in navigating the system.

"'Back then,' fathers were expected to wait down the hall during delivery, and if they were allowed in, they were expected to stay out of the way and watch. Now they can be invited into the delivery room and may even cut the umbilical cord," he said. "But the keyword here is invited. Men familiar with the traditional viewpoint around childbirth may feel pregnancy and childbirth is a womenowned realm and may feel they need permission to participate."

\section{"Williams has conducted focus groups with fathers, where they tell him they want to be involved with the children at the outset, but that they also needed help in navigating the system."}

This includes involvement from the outset, in prenatal meetings all the way through the birthing process and maternal and infant support through the first year of life and beyond.

"If they have brought the mother in for her prenatal check-up but are sitting in the waiting room, ask them if they would like to join the meeting. And then talk to them when they are there and enlist their partnership," Williams said.

"For instance, the number one reason women stop breastfeeding is they think they are not making enough milk for the baby because the infant seems constantly hungry," he said. "But we know it is because a newborn baby's stomach is the size of a cherry. $A$ father can help reassure the mother that she is doing everything all right. He can be a source of strength and protection for her during both delivery and the post-natal period, by understanding what is happening and what she and the baby need. And he is there to claim his own dad and baby feeding and bonding time."

Williams also leads Daddies' Café, supported by the WIC Division of the Detroit Health Department. The all-male meetings include a discussion on infant safe sleep and a presentation on breastfeeding basics, as well as open discussion.

"Until you learn more about breast milk, you believe what you hear in ads that say formula is the next best thing," Williams said. "Since a dad's primary responsibility is to protect and provide, when dads know more about breastfeeding, they are better equipped to assist from day one."

Williams notes that what the fathers his program works with come to understand is straightforward:

- You and your opinions matter.
Your baby deserves the health benefits of breastfeeding.

- Mom is more likely to breastfeed and do so for longer, with your support.

- $\quad$ You are in a position to protect the skin-to-skin, nursing, and bonding space for mom at both the hospital and at home.

- You should also make time for your own skin-to-skin contact, nursing, and bonding time.

\section{The Role of the Health Care Provider in Recognizing Bias}

Since the gatekeeper for access to paternal involvement may be health care providers, it is important for the pre- and perinatal health care community to:

- Recognize and understand your own biases. (For example, Teaching Tolerance offers a self-test on hidden bias.) (5)

- Determine if you have misconceptions about dad's role and his role at the hospital.

- $\quad$ Reflect on men's wants and needs in this process.

- Be careful not to automatically marginalize or exclude them from participation.

- Understand that men might not always say upfront what they want.

We know from our work with Peter Williams and other colleagues who are doing paternal outreach, and from the barriers we uncover in our Straight Talk for Infant Safe Sleep, that empathy and inclusion for fathers and partners are central factors to gaining acceptance of infant safe sleep and breastfeeding practices, and therefore central to reducing infant mortality in the first year of life.

Information on First Candle and its bereavement support services can be found on the First Candle website. The Grief Support Line is $1-800-221-7437$.

\section{References:}

1. Overview: Paternal Involvement and Pregnancy Outcomes, NICHD Fatherhood Outreach Meeting, July 28-29, 2015. Presentation.

2. Two Months of Breastfeeding Cuts SIDS Risk in Half: University of Virginia Health System Physician Resource. https://www.uvaphysicianresource.com/sids/

3. Dads make a difference: an exploratory study of paternal support for breastfeeding in Perth, Western Australia. International Breastfeeding Journal, 29 November 2009. https:// internationalbreastfeedingiournal.biomedcentral.com/articles/10.1186/1746-4358-4-15

4. Cited in Breastfeeding Connections, July/August 2019, Michigan Department of Health and Human Services, WIC Division. https://www.michigan.gov/documents/mdhhs/BF Connections July August 662102 7.pdf

5. Teaching Tolerance. http://www.tolerance.org/supplement/testyourself-hidden-bias

Disclosure: The author is the Director of Education and 
Bereavement Services of First Candle, Inc., a Connecticut not for profit 501c3 corporation.

NT

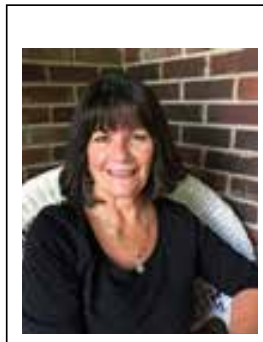

Corresponding Author

Barb Himes, IBCLC

Director of Education and Bereavement Services

First Candle

49 Locust Avenue, Suite 104

New Canaan CT 06840

Telephone: 1-203-966-1300

For Grief Support: 1-800-221-7437

barb@firstcandle.org www. firstcandle.org

\section{Still a Preemie?}

Some preemies are born months early, at extremely low

birthweights. They fight for each breath and face nearly

insurmountable health obstacles.

But that's not every preemie's story.

Born between

34 and 36 weeks gestation?

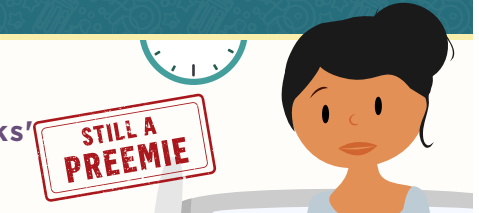

Just like preemies born much earlier, these "late preterm" infants can face:

$\stackrel{9}{9}$
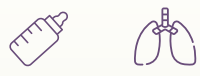

Jaundice Feeding issues

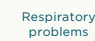

And their parents, like all parents

of preemies, are at risk for

postpartum depression and PTSD.

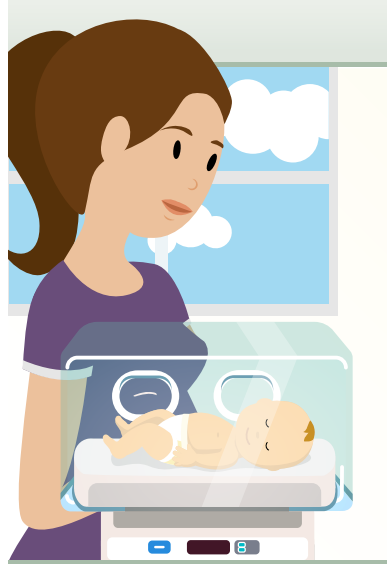

Born preterm at a "normal"
weight?

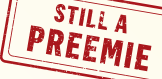

Though these babies look healthy, they can still have complications and require NICU care.

But because some health plans determine coverage based on preemie's weight, families of babies that weigh more may face access barriers and @NEOTODAY

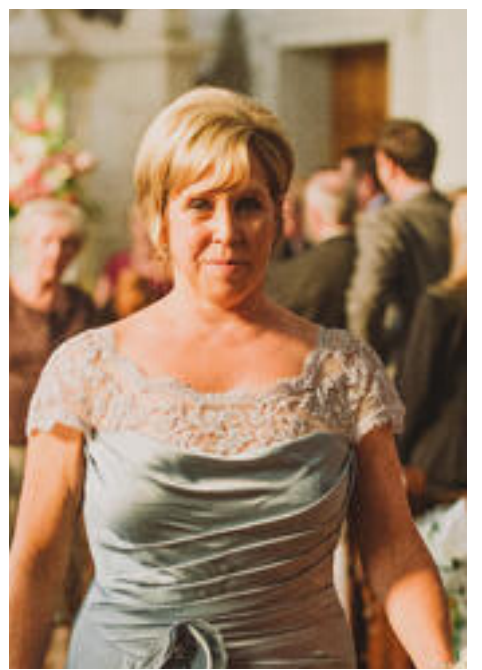

In January, heaven gained a new angel -

Laura Reno.

Laura was a SIDS mom and a guiding force at First Candle.

She worked tirelessly to end SIDS and was a source of comfort for many of our berieved families.

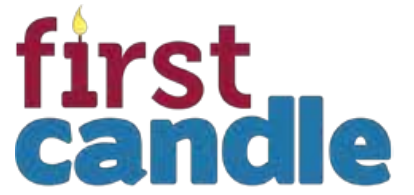

Laura will be greatly missed.
Born preterm but not admitted to the NICU?

Even if preterm babies don't require NICU care, they can still face health challenges. Those challenges can extend through childhood, adolescence and even into adulthood.

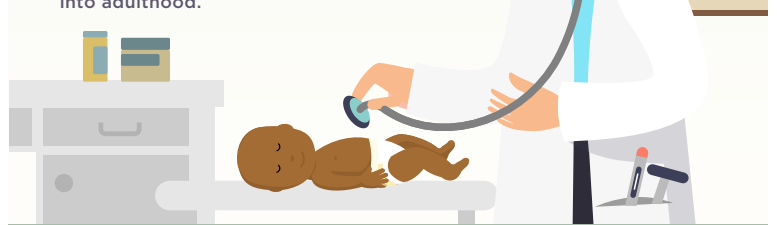

Some Preemies

Will spend weeks

in the hospital

W. Will have lifelong

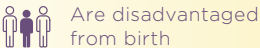

All Preemies

ङ Face health

$\nrightarrow$ Deserve appropriate

health coverage

Qo Need access to www.infanthealth.org

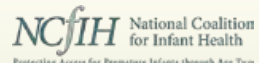

Saving babies. Supporting families 